Cita bibliográfica: García-Domingo, M. (2019). Reflexión profesional sobre la realidad de la intervención social: retrocesos propios de un periodo de postcrisis y propuestas de mejora. [Professional reflection about the reality of social intervention: setbacks of a post-crisis period and improvement proposals]. Alternativas. Cuadernos de Trabajo Social, 26, 103-122. https://doi.org/10.14198/ALTERN2019.26.05

\title{
REFLEXIÓN PROFESIONAL SOBRE LA REALIDAD DE LA INTERVENCIÓN SOCIAL: RETROCESOS PROPIOS DE UN PERIODO DE POSTCRISIS Y PROPUESTAS DE MEJORA
}

\section{PROFESSIONAL REFLECTION ABOUT THE REALITY OF SOCIAL INTERVENTION: SETBACKS OF A POST-CRISIS PERIOD AND IMPROVEMENT PROPOSALS}

\author{
MARTA GARCÍA-DOMINGO (iD \\ Departamento de Psicología (Área de Trabajo Social y Servicios Sociales) \\ Universidad de Jaén. Paraje las Lagunillas s/n, Edicifio C5-148. 23071 Jaén (España) \\ Correo:mgdoming@ujaen.es
}

\begin{abstract}
Resumen
Inmersos en la antesala de una nueva crisis socioeconómica, los efectos y consecuencias de la anterior crisis son aun notorios en el sistema de servicios sociales español, percibiéndose importantes limitaciones en la práctica profesional del Trabajo Social. Nos aproximamos a esta realidad desde una metodología cualitativa, basada en la técnica de la entrevista, con la pretensión de dar voz a las reflexiones de las/os trabajadoras sociales y valor a sus planteamientos. Encontramos propuestas de mejora a distintos niveles de intervención: (1) a nivel individual, familiar y grupal se defiende el acompañamiento y apoyo subsidiario, el empoderamiento y el refuerzo de la visión humanitaria, entre otros; (2) a nivel estratégico, se subraya la importancia de la coordinación interinstitucional, la cohesión y la participación social; y (3) a nivel holístico, se apuesta por una sociedad más inclusiva y diversa, que reconozca distintos estilos de vida y desarrolle una concepción más social del éxito.
\end{abstract}

Palabras clave: Propuestas de mejora, intervención social, Trabajo Social, Post-crisis, Pre-crisis, servicios sociales.

\begin{abstract}
On the cusp of a new socioeconomic crisis, the impact of Spain's previous crisis is still noticeable in the country's system of social services and significant limitations can be observed in Social Work practice. We approach this reality adopting a qualitative methodology based on interviews with Social Workers, thus giving a voice to their reflections and a value to their approaches. Improvement proposals were found at different levels: (1) at an individual, family and group level of intervention, defending accompaniment and subsidiary support, and through the empowerment and strengthening of a humanitarian vision, among others; (2) at a strategic level, emphasizing the key role of inter-institutional coordination, social cohesion and participation; and (3) on a holistic level, a commitment to a more inclusive and diverse society, which acknowledges different lifestyles and develops a more socially-oriented conception of success.
\end{abstract}

Keywords: Improvement proposals, social intervention, Social Work, Post-crisis, Pre-crisis, social services. 


\section{Extended abstract}

EU countries are undergoing a supranational convergence process that defines them, causing a true restructuring of their policies. The present article reveals that neoliberal ideas and policies, as well as post crisis consequences, determine social intervention in the EU, generally, and in the Spanish context, in particular. Experts and social analysts have pointed to the current tendency for social development to be contingent on economic development, if not replaced by it (Vicente-Giménez and Berzosa, 2014). Public social services and third sector organizations have been forced to redefine themselves in recent years. Moreover, the effect of this trend on Social Work is unquestionable, due to the cause-effect link between the historical, political, economic context and the development of the profession (Abad and Martín, 2015).

Previous studies have indicated changes in the characteristics of social services and social intervention, among which: its increasingly restrictive nature, i.e. a system marked by restrictions, limits, control and an overly bureaucratic approach, leaving many situations and needs unattended (García-Domingo, 2018); social privatization (Alguacil, 2012); a regression in social policies inspiring principles and purpose, and imbued with a charitable-assistance character (Ioakimidis, Cruz and Martínez, 2014); a greater presence of the social control paradigm (Peláez and Pastor, 2019); naturalization of problems and a decreased sense of public responsibility (Ávila and Malo de Molina, 2010); poor strategic planning in social organisations and negative perception of Social Work (Pacheco-Mangas and Palma-García, 2015); time limits for social intervention (Ballestero, Úriz and Viscarret, 2012); an increase in social volunteering by professionals and a decline in corporatism (Barrera-Algarín, Malagón-Bernal and Sarasola-Sánchez, 2013). All this entails a loss of autonomy and identity status in active social workers (Morley and Dunstan, 2013) and in social work students (García-Moreno and Anleu-Hernández, 2019). This critical situation presents a challenge for Social Work that must be undertaken proactively. In recent years, a large number of authors have claimed the need to understand the crisis as a challenge, struggling against the pessimistic view of a society hardly hit by the crisis (García-Domingo and Sotomayor, 2017).

Therefore, various authors have revealed a return to formulas of attention proper to previous stages (social assistance), although few studies have hitherto pointed to a possible radicalization of this trend as a result of the new crisis. Furthermore, few studies have used the knowledge and experience of social workers to make improvement proposals based on their analysis of the situation in the light of this paradigm shift.

Methodology: The present research focused on the importance of involving social workers in the analysis of today's social services reality and in making improvement proposals. We approached this reality using a qualitative methodology, which gave a voice to Social Workers' reflections and a value to their proposals. Not only were their proposals essential, but the reflections and arguments used to defend them were of major relevance.

Results and discussion: Proposals at different levels were found: 1. Regarding social intervention: focused on the person; accompaniment; community intervention. 2. At a strategic and organisational level: social participation and cohesion. 3. Implementation or consolidation of social changes: a more inclusive society with a conception of success that is more socially-oriented.

Conclusions: Spain's Social Services system requires changes and improvements at different levels. Giving a voice to social workers' reflections and proposals is key to this progress. 


\section{Introducción}

La mayoría de académicos/as coinciden en apuntar los notorios efectos experimentados en materia de bienestar social como consecuencia de la última crisis económica, hasta el punto de denominarla crisis social. No obstante, no hay consenso a la hora de identificar las consecuencias de la última crisis económica internacional en el presente y futuro de los Estados de Bienestar europeos (Szikra, 2014). Sin haberse podido llegar a un consenso con respecto al efecto de la crisis de 2008 en los Estados de Bienestar, los economistas señalan que nos estamos adentrando ya en otra crisis socioeconómica, de la que cabe esperar un re-agravamiento de la precarización de la «clase media».

Por una parte, está la tesis, defendida por multitud de autores/as, de que los acontecimientos acaecidos a partir de 2008 han cuestionado los propios fundamentos de los sistemas de protección social a través de una serie de reformas paradigmáticas. Este proceso podría poner fin a la ambición de crear una Europa social (Pochet y Degryse, 2012; Taylor-Gooby, 2012). Sin embargo, una minoría de autores como Starke, Kaash y Van-Hooren (2013) defienden la inexistencia de un patrón consistente de reducción de gastos de bienestar social o austeridad, encontrándose las diferencias realmente significativas en las intervenciones según las regiones y el tamaño de los Estados de Bienestar. Por otro lado, tampoco hay conformidad en señalar a la crisis como factor desencadenante primordial de los cambios experimentados en materia de protección social, ya que son muchos los que apuntan a que dichos cambios son consecuencia de la penetración de ideas neolibearales, del «triunfo» del mundo del capital -que supone el debilitamiento del mercado de trabajo, de los salarios y del Estado de Bienestar-, así como de la utilización de la globalización económica como argumento para romper el pacto y frenar el avance social y laboral (Navarro, 2014). En lo que sí coinciden las personas expertas y analistas es en apuntar la actual tendencia a que el desarrollo de lo social quede supeditado, si no sustituido, por el desarrollo económico. Como afirman Vicente-Giménez y Berzosa (2014):

(...) en nuestros días, los valores, la ética, el derecho y la política, esto es, el espacio jurídico-político cede su lugar a la hegemonía de la economía, y con ello, la democracia occidental se supedita a los grandes intereses de un modelo económico que se impone como estructura básica de nuestra sociedad, única e inamovible (p. 13).

Siendo el Trabajo Social una profesión fuertemente vinculada al desarrollo de los Estados de Bienestar, cabe esperar que la época dorada del estado de bienestar coincida con su despliegue y consolidación. Asimismo, no es de extrañar que el retroceso de los derechos sociales, los estados de bienestar y 
la incorporación de lógicas mercantilistas, venga acompañada de una crisis de la profesión. Partimos en el presente estudio de la premisa de que resulta fundamental analizar el impacto de este repliegue social en la intervención desarrollada desde el Trabajo Social. Lo hacemos en una época de postcrisis, en la que los efectos se podrían percibir como superados, si bien resulta difícil dejarlos atrás en un contexto que ya apunta al adentramiento en una nueva situación de crisis socioeconómica.

Tal y como destacan Abad y Martín (2015), la naturaleza histórico-social del Trabajo Social implica su condicionamiento a las coordenadas de tiempo y espacio que van reconfigurando y resignificando sus elementos de base. La veracidad de esta afirmación ha sido constatada por los estudios que han profundizado en la realidad del Trabajo Social en el contexto actual. Autoras como García-Domingo (2018) apuntan al carácter crecientemente restrictivo, burocrático y controlador del sistema público de servicios sociales de la crisis y postcrisis, así como a la privatización y concertación de servicios sociales (Alguacil, 2012) como estrategia de reducción de costes. Otros estudios reflejan también la transformación de la práctica del Trabajo Social a fórmulas caritativas en la última década, lo que cuestiona los principios inspiradores básicos de nuestra profesión como el derecho a la dignidad y la justicia social (Ioakimidis, Cruz y Martínez, 2014). Peláez y Pastor (2019), centrando la atención en el caso andaluz, identifican prácticas próximas a la beneficencia en el que determinados recursos sociales se impregnan del paradigma de control social con una fuerte estigmatización asociada, especialmente en el sector rural. En este panorama de expansión de la precariedad, se naturalizan los problemas y disminuye el sentido de responsabilidad pública al ser considerados estos como fenómenos inevitables (Ávila y Malo de Molina, 2010).

Las/os profesionales del Trabajo Social se enfrentan así a nuevos desafíos y dilemas éticos en su desempeño laboral, entre los que Ballestero, Úriz y Viscarret (2012) subrayan los siguientes: la creciente burocratización disminuye los tiempos de las intervenciones, presentándose a menudo la disyuntiva de cerrar un expediente por la presión existente sin tener la seguridad de que su cierre sea beneficioso; se plantean también dilemas con respecto a la distribución de los recursos limitados, surgiendo dificultades a la hora de establecer criterios distributivos justos. En definitiva, se pone de manifiesto la vuelta a modelos basados en el asistencialismo y la beneficencia, más centrados en la gestión de prestaciones básicas que en intervenciones basadas en fórmulas más propias del Trabajo Social de la primera década del siglo XXI: trabajo comunitario, participación ciudadana, prevención y promoción social, empoderamiento, acompañamiento social, entre otros (Uceda, Martínez, Navarro y Botija, 2014). 
Ejemplo de ello son las políticas de rentas mínimas que, si bien se sustentan en la retórica de la inclusión y la seguridad universal, en la práctica ponen de manifiesto la realidad de privación material severa y exclusión absoluta de un porcentaje alto de la población, así como del estigma asociado a su percepción. En su estudio, Pacheco-Mangas y Palma-García (2015), reflejan que las/os profesionales perciben deficiencias en cuanto a visión y misión de las organizaciones sociales en las que trabajan, así como una planificación estratégica inadecuada para el abordaje eficaz de las problemáticas sociales presentes, lo que no solo frustra las pretensiones originales de la profesión, sino que además va en detrimento de la visión exterior de la práctica profesional de la misma. Detectan, asimismo, que las/os profesionales se quejan de una formación de calidad insuficiente para hacer frente a su quehacer diario. En relación a esto, Morley y Dunstan (2013) afirman que se devalúan las habilidades y el conocimiento de las/os profesionales del Trabajo Social, su autonomía y su identidad. Esto afecta no solo a las/os profesionales en activo, sino también a sus estudiantes, que ven como el Trabajo Social se aleja de sus postulados de principios del siglo XXI (García-Moreno y Anleu-Hernández, 2019). Por su parte, Barrera-Algarín, Malagón-Bernal y Sarasola-Sánchez (2013), reflejan los siguientes cambios en el Trabajo Social como consecuencia de la deconstrucción de los Estados de Bienestar: las condiciones cada vez más precarias de las/ os profesionales del Trabajo Social; un alejamiento metodológico del paradigma conflictual y un auge del funcionalismo, más propio de corrientes neoliberales; un aumento del voluntariado social desempeñado por profesionales, en detrimento del desarrollo de la profesión; y un declive del corporativismo, que se aleja del panorama de inicio de la crisis en el que surgieron iniciativas corporativistas como la Marea Naranja, protagonizada por las/os profesionales del Trabajo Social. En los últimos años nos encontramos con un fuerte cuestionamiento social de la profesión que encuentra una oposición y respuesta corporativa fuerte a nivel cualitativo y argumental, pero insuficiente cuantitativamente debido al bajo porcentaje de profesionales colegiados.

En contraposición a la perspectiva pesimista anterior, encontramos estudios que reflejan que en contextos de crisis se produce una mayor polarización de las diferentes figuras del Trabajo Social, hallándose así la figura del trabajador/a social «crítico»o «comprometido», que cuestiona la respuesta limitada impuesta en situaciones de crisis y asume una intervención centrada en la persona y su situación en un sentido holístico (Gaspar, 2012; Abad y Martín, 2015). Dicho de otro modo, lo que para unos se presenta como un panorama desolador y frustrante, para otros plantea retos, canalizados en un rol proactivo a través del que se plantean reivindicaciones, propuestas y mejoras 
en sus principales áreas de influencia (García-Domingo y Sotomayor, 2017). En definitiva, se parte de las consecuencias propias de los contextos adversos como elementos impulsores del cambio. En este punto, resulta fundamental difundir entre las/os profesionales la necesidad de cuidarse para cuidar, avalada por Puig (2015) en las siguientes diez propuestas teórico-prácticas: interdependencia, inteligencia colectiva, doble cuidado, atención a la subjetividad, dotarse de una mirada molecular sobre las situaciones, confianza, volver a la palabra, creatividad y cuidado, investigar para cuidar y cuidarse y, por último, supervisión social. Solo en una situación de autocuidado y equilibrio personal y profesional, las trabajadoras/es sociales podrán confrontar los retrocesos sociales experimentados y liderar propuestas de mejoras eficaces y sostenibles.

Tenido todo lo anterior en cuenta, constatamos que son cuantiosas las investigaciones que ponen de manifiesto las consecuencias de la crisis en la precarización de la sociedad española, siendo también numerosas, aunque en menor medida, las investigaciones científicas que han aportado luz sobre el impacto de esta realidad en nuestra profesión. Sin embargo, son pocos los análisis crítico-propositivos de esta realidad que, a tenor de la percepción de las personas que desarrollan la atención e intervención profesional frente a las situaciones de necesidad sociales, avancen en el conocimiento de los cambios pertinentes. Además, cuando se habla de los efectos sociales de la crisis no se analiza hasta qué punto se puede hablar de una situación de estancamiento o carácter estructural del impacto de la crisis en los sistemas de servicios sociales, que suponga un retroceso de los mismos incluso en situaciones de mayor prosperidad económica. En definitiva, el actual escenario de la intervención requiere de un sobreesfuerzo de las/os profesionales de lo social por prestar atención no solo a lo urgente, sino también, y, sobre todo, a lo importante.

Asimismo, resulta fundamental que los poderes públicos tengan en cuenta la realidad actual de los servicios sociales y pongan a disposición de los mismos las medidas necesarias para mitigar la situación de riesgo, que afecta ya incluso a la formación académica de las/os futuras/os profesionales del Trabajo Social, cuyos postulados se ven tambaleados por una situación de crisis que parece estancarse.

\section{Metodología}

En la presente investigación pretendemos dar voz a las reflexiones de las/os profesionales de servicios sociales sobre la realidad del sistema, los cambios experimentados y los riesgos aparejados, así como plantear alternativas a las debilidades identificadas. Para ello se ha empleado una metodología cualitativa, a partir de la entrevista como técnica de conocimiento, que destaca por 
sus múltiples aplicaciones en el campo de las Ciencias Sociales, así como por la multitud de propósitos y finalidades para la que puede ser empleada. En este sentido:

(...) la entrevista nace de una ignorancia consciente por parte del entrevistador quien, lejos de suponer que conoce, a través de su comportamiento exterior, el sentido que los individuos dan a sus actos, se compromete a preguntárselo a los interesados, de tal modo que éstos puedan expresarlo en sus propios términos y con la suficiente profundidad para captar toda la riqueza de su significado (Ruiz- Olabuénaga, 1996, p. 171).

Partimos, por tanto, de la premisa de que la realidad social y el comportamiento humano no pueden entenderse sin indagar en los significados y propósitos que los/as actores/as adjudican a sus actividades (Guba y Lincoln, 2002). Para abordar nuestro propósito, se entrevistó a un total de 19 profesionales de Trabajo Social del contexto español, seleccionados/as a través de muestreo intencional no probabilístico, determinándose la muestra por saturación. La elección de las personas participantes se hizo atendiendo a cuestiones como su trayectoria profesional, su pertenencia a distintos ámbitos (público, privado y tercer sector) y niveles de intervención (primario y especializado), así como su grado de participación e implicación en pro del avance de la profesión, entre otros. Destaca la prolongada trayectoria profesional de la mayoría de las/os participantes, que les permitió adentrarse en dicha reflexión desde una perspectiva longitudinal, así como vislumbrar los cambios en la última década. El alto grado de profundidad alcanzado en la mayoría de las entrevistas y el bajo nivel de estructuración de las mismas permiten que, de manera espontánea y en diferentes momentos de la entrevista, a través de la reflexión y argumentación, surjan, de manera directa e indirecta, propuestas de mejora de gran pertinencia. En el presente análisis exploratorio, se abordan dichas propuestas contextualizadas en una situación de postcrisis en la que resulta fundamental reflexionar sobre las actuales tendencias de la intervención social y sus riesgos, confrontándolos con los principios y el objeto de la profesión. De gran valor son, no solo las propuestas, sino la manera de argumentarlas y las reflexiones que subyacen a las mismas.

Por otro lado, la participación de profesionales pertenecientes al ámbito público y al tercer sector, así como a servicios sociales comunitarios y especializados, permite tener una visión más holística y, a su vez, realizar una comparativa sobre las propuestas realizadas en ambos ámbitos y niveles de intervención.

Las entrevistas tuvieron lugar en despachos de las personas entrevistadas, con la única presencia de entrevistadora y entrevistada/o, previa exposición 
por parte de la primera de los objetivos de la investigación y explotación de los datos obtenidos. Las mismas fueron grabadas, transcritas íntegramente, codificadas, categorizadas y analizadas, con la ayuda del programa Atlas.Ti (versión 8), realizándose un análisis de contenido de las mismas, desde un enfoque evolutivo y tendencial. Codificamos las entrevistas con una letra inicial, que hace referencia al perfil profesional genérico de las/os participantes $(\mathrm{P}=$ Profesionales del Trabajo Social $)$. La segunda letra refiere el ámbito de actuación ( $\mathrm{p}=$ público; $\mathrm{t}=$ tercer sector). La tercera letra se corresponde con el nivel de intervención ( $c=$ comunitario; $\mathrm{e}=$ especializado). Por último, un número identificativo de la entrevista, que responde al orden de realización de la misma.

\section{Resultados y discusión}

3.1. Propuestas de mejora en la intervención social: afianzando el Trabajo Social individual, familiar, grupal y comunitario

En oposición a las exigencias de la práctica profesional actual, que como ha quedado reflejada en la introducción se caracteriza por los tiempos de intervención reducidos y la burocratización, las personas informantes justifican la necesidad de una intervención (más) centrada en el individuo y su familia. Para ello resulta fundamental partir de un estudio exhaustivo de su problemática y necesidades, que aportará claves fundamentales para la posterior intervención. Esta propuesta encuentra su sentido en la necesidad de superar el carácter asistencialista de las políticas de antaño que, como ya hemos mencionado, ha recobrado importancia como resultado de la última crisis.

«Por ejemplo, ahora mismo estamos poniendo en marcha un programa a nivel nacional que está funcionando desde hace tiempo en Madrid y Barcelona y que estamos implantando aquí, lo que nos va a permitir tener un conocimiento global de la problemática de todas y cada una de las familias que atendemos y, en consecuencia, eso nos va a permitir la posibilidad de intervenir con ellos en el planteamiento del futuro de esa familia. No es lo mismo acoger a una persona que nos diga 'no tengo que comer' y decirle 'tenga usted una bolsa de comida' y se ha acabado, que hacerle un DAFO a cada familia, estudiarlas y como consecuencia de eso aconsejarles» (P.p.c.8)

Para ello y como ha sido constatado por la literatura previa, resulta fundamental una atención centrada en la persona y sus fortalezas (Saleebey, 1996; Zimmerman, 2006), en lugar de centrada en sus déficits y carencias, que no hace más que reforzar su vulnerabilidad y la relación de poder desigual entre profesional del Trabajo Social y persona usuaria (De la Paz, Rodríguez y Mercado, 2014). Sin embargo, la atención individualizada no puede constituir 
la única estrategia de intervención a desarrollar por el Trabajo Social, sino que también se aboga por el desarrollo de proyectos específicos para grupos de personas con problemáticas similares que, a su vez, desarrollen una labor centrada en el individuo. En este sentido, ponen de manifiesto la reducción de proyectos de intervención grupales puestos en marcha desde el ámbito público, fruto de las restricciones administrativas propias de la última década. Autores como Antón (2012) señalan esta tendencia hacia la «asistencialización» y control del «consumo», puesta de manifiesto en la reducción de la cobertura protectora de políticas sociales como las rentas mínimas o medidas de integración sociolaboral, que lejos de permitir una intervención centrada en el individuo y su contexto, se tornan in instrumentos selectivos de acceso a los recursos sociales.

En este sentido, las/os informantes apuntan la necesidad de apostar en mayor medida por el desarrollo de proyectos grupales flexibles de respuesta a necesidades y demandas presentes en los contextos objeto de intervención que, sin embargo, no tienen cabida en otro tipo de programas y servicios por las restricciones en los requisitos de acceso.

«Y yo creo que necesitamos más proyectos grupales especializados de este tipo para apoyar a los casos individuales. Nosotros tenemos que seguir las reglas y alguna gente puede ajustarse a las reglas, pero otros no. Así que debemos hacer más proyectos centrados en el individuo» (P.t.e.4)

Así, defienden la importancia de involucrar en mayor medida a las/os técnicos en el proceso de diseño de los planes, programas y proyectos de intervención, cuya perspectiva orientará actuaciones más holísticas que superen a la mera prestación económica o de servicios. Esta necesidad de reconocer la figura del profesional del Trabajo Social como figura de enlace con capacidad de influir en los puestos de responsabilidad y toma de decisiones ya ha sido señalada por autores como García y Arévalo (2016), que reivindican el reconocimiento del tiempo invertido por las/os trabajadoras/es sociales en funciones vinculadas a la creación de redes, apoyo a grupos y creación/mantenimiento de nuevos recursos, entre otros, superando la percepción limitada y reduccionista de la profesión asociada a la mera gestión de recursos.

«Está visto que aquellas medidas o aquellas políticas sociales que van destinadas o que tienen un planteamiento orientado a desarrollar un plan de intervención, a trabajar más el tema de la intervención y con la iniciativa de los propios técnicos, funcionan mejor que cuando a ti te dan un programa desde instancias político administrativas superiores y se orienta más a una prestación económica, y tiende a desvirtuarse» (P.t.e.7) 
Se evidencia, por tanto, el reclamo de una mayor incorporación de la figura profesional en los Consejos Asesores y grupos de expertos conformados, promoviendo funciones de asesoramiento y consultoría en la elaboración y modificación de leyes, decretos, planes y programas vinculados a la acción social, los servicios sociales y las políticas sociales (Consejo General del Trabajo Social, 2019).

Sin embargo, esta no es la única función profesional a fortalecer. El «acompañamiento» es uno de los principales elementos de mejora propuestos por las/ os propios técnicos en el actual contexto crítico, demandándose la necesidad de poner a disposición de los centros sociales los recursos que posibiliten un acompañamiento real, efectivo y sentido por las propias personas usuarias.

«(...) un acompañamiento a la familia nos lleva a poder cambiarle las actitudes en cuanto al futuro de esa familia, y haciendo ese acompañamiento con afectividad y agrado para que empiece a tener esperanza, ya que si no se les hace ese acompañamiento es muy difícil dentro de la situación que están sufriendo» (P.p.c.8)

Como destaca el Consejo General del Trabajo Social (2019) el apoyo emocional y social son campos de competencia de las/os profesionales del Trabajo Social, defendiéndose, en esta línea, la importancia de incrementar el número de horas dedicadas al acompañamiento y reducir las de despacho, prestando una mayor atención al desarrollo de destrezas por parte de las dos partes implicadas (Smale, Tuson y Statham, 2015). Cabe destacar que, en el presente análisis, encontramos que las/os trabajadoras/es sociales de instituciones del tercer sector proclaman, en mayor medida que las/os profesionales del ámbito público, la importancia de que, incluso si no hay recursos que ofrecer a la persona que viene a solicitar ayuda, se le preste una especie de apoyo subsidiario a través de la atención y el acompañamiento personalizado. Coinciden en destacar la insuficiente personalización en la atención que, a menudo, se presta a las personas usuarias desde el sector público, reflejando una tendencia a la deshumanización. En contraposición a ello, destacan los beneficios de la proximidad y cercanía.

«La acogida de una persona que toca a la puerta y sonreirle, el estilo y los modos de hacer... eso tiene una enorme transcendencia. Yo tengo la experiencia de haber estado con una persona tres cuartos de hora y al final le dije que lamentaba no poder ayudarle y me dijo, "no importa yo he sido atendido y jamás nadie me había escuchado del modo que lo habéis hecho vosotros». Quiere decir que el ser humano tiene cosas mucho más valiosas que lo exclusivamente material, que es una necesidad imprescindible para sobrevivir, pero el interior del ser humano es mucho más valioso» (P.t.e.14) 
En relación con lo anterior, inciden en la importancia de reforzar la visión humanitaria por parte de las/os técnicos, que corren el riesgo de una tecnificación excesiva:

«Somos demasiado técnicos en la forma de atajar los problemas e incluso de ver a la gente. Probablemente hay una falta de ideología. Pero tendríamos que encontrar a la persona. Necesitaríamos reforzar la visión humanitaria» (P.t.e.19)

Además, recuerdan que los agentes sociales, especialmente en un contexto como el actual en el que los recursos son limitados, no tienen la función de buscar la solución a los problemas que llegan a su oficina, sino buscar con la familia una salida de futuro y otorgarle las claves para que puedan solventar las contingencias. En la misma línea, muestran la necesidad de un cambio de perspectiva en la que el profesional tenga más en cuenta el punto de vista de la persona usuaria en la definición del problema y búsqueda de soluciones. Esto implica abandonar el carácter paternalista que, en algunos casos, sigue latente en cierta medida en las profesiones sociales. Es imprescindible, por tanto, que las/os técnicos sociales se involucren en la agenda pública como expertas/os en los problemas que se abordan, pero también que tengan en cuenta la perspectiva de aquellas personas que experimentan dicha problemática, fomentando su empoderamiento y participación en la definición de sus problemas y posibilidades de mejora.

«Nos está costando cambiar la perspectiva y tener más en cuenta su punto de vista. Nosotros no somos los expertos/as, lo son ellos, porque son los que tienen la experiencia» (P.p.c.11)

Por otro lado, apuntan dos ideas clave: la importancia de una intervención temprana y de fomentar el Trabajo Social Comunitario. Con respecto a la intervención temprana:

"Cuando la gente empieza a no poder pagar el alquiler, en el primer mes, los servicios sociales deberían involucrarse. Asi podrían evitarse muchos problemas. Se trata de intervenir antes de que la gente sea expulsada de sus casas» (P.p.e.3)

Como se extrae de la lectura del fragmento anterior, resulta conveniente una detección temprana del problema que permita una pronta intervención, evitándose futuras situaciones de riesgo más difíciles de atajar. Este reto cobra especial sentido en referencia a los nuevos colectivos en riesgo, que a menudo por desconocimiento, falta de costumbre o prejuicios, no acuden a los servicios de bienestar o lo hacen demasiado tarde, cuando la situación es difícil de revertir y los problemas asociados se han acentuado.

Con respecto a la segunda, ponen de manifiesto la importancia de reestablecer el Trabajo Social Comunitario, que en los últimos años podría estar 
perdiendo peso. La defensa de una «sociabilidad emancipadora», generadora de «redes que dan libertad» (Riechman y Fernández-Buey, 1994) que busca soluciones consensuadas a conflictos relevantes (Habermas, 1998) no resulta novedosa. Sin embargo, su recuperación en clave democrática ha resurgido en la última década, vinculada al Trabajo Social dada su posición estratégica, otorgándole un papel protagonista tanto en la definición como en el desarrollo del proceso movilizador de agentes y recursos comunitarios (Telleira, 2010). Esto cobra especial sentido si se tiene en cuenta que el trabajo en y con comunidades es patrimonio del Trabajo Social, que fue la profesión pionera en su implantación, a la que posteriormente se le fueron sumando otras (Marchioni, 1999). Destacan, en definitiva, la importancia de trabajar con el tejido social a nivel comunitario y dar respuesta a demandas colectivas, ampliamente reconocido pero que aún cuenta con insuficientes experiencias prácticas. Un ejemplo de práctica comunitaria, en el contexto andaluz, es la Estrategia Regional Andaluza para la Cohesión e Inclusión Social (ERACIS), de intervención en zonas desfavorecidas, de la que se esperan importantes avances a nivel comunitario. En definitiva, las/os expertas/os del presente estudio argumentan que la importancia de centrarnos en el individuo (enfoque centrado en las personas) no contradice ni entra en conflicto con un énfasis en lo colectivo (identificación de nuevos colectivos en riesgo y de colectivos estructuralmente vulnerables), que permita divisar los problemas de la sociedad en su conjunto, y de una intervención comunitaria:

«Por último, no sólo lo individual, sino lo colectivo. Tenemos que hablar sobre esto, porque lo que se ve es que están aumentando los problemas de la gente... Incluso entre los que tienen un trabajo e ingresos altos. Las tasas de enfermedades psiquiátricas no dejan de crecer en estos niveles, lo que significa que la vida que viven no es buena, y eso que tienen un nivel de vida alto, pero no es una buena vida» (P.p.e.16)

3.2. Propuestas a nivel estratégico y de organización: la importancia de la cohesión y participación sociales

Como propuestas que podríamos ubicar en un nivel estratégico y organizativo, las/os participantes destacan la coordinación, referida a dos cuestiones clave. Por un lado, la conveniencia de una coordinación entre los distintos ámbitos y niveles profesionales de intervención.

«Yo creo que es muy importante conectar el nivel académico, científico con el institucional y político» (P.p.c.1).

Por otro lado, una más efectiva coordinación entre los distintos países de la Unión Europea, que genere mayores niveles de cohesión social intracomunitaria: 
"Creo que necesitamos una mejor coordinación de políticas entre países de la Unión Europea, más intercambio entre países. Si trabajamos juntos, podemos fortalecernos. Al menos eso pienso yo» (P.t.e.14)

Si bien las/os profesionales entrevistadas/os realizan una enérgica defensa de la responsabilidad pública y el sistema público de protección y promoción social, en menor medida $-\mathrm{y}$ sin entrar en contradicción con dicha defensa- también se apuesta por el fomento del asociacionismo y la participación ciudadana. En esta línea, De la Red y Barranco (2014) recogen el doble beneficio asociado a los mayores niveles de participación social: por un lado, da lugar a una mayor riqueza de recursos humanos y redes de apoyo disponibles para dar respuesta a los problemas y necesidades sociales; por otro, favorece el desarrollo del tejido social, la justicia y la equidad social.

"Trataría de fomentar y fortalecer a los grupos pequeños que hay a través de apoyo financiero a estos grupos, ONGS u organizaciones que apoyan a los grupos o colectivos en riesgo» (P.p.c.13)

En España, con la crisis y el cuestionamiento del modelo público burocrático garantista, nos encontramos con una creciente incorporación del mercado a la cuestión social, que parece sacar provecho de la creciente desconfianza de la sociedad civil en las instituciones sociales tradicionales, así como del desprestigio político (Alguacil, 2012; Prats, 2005). Así pues, confluyen en el panorama social instituciones privadas con y sin ánimo de lucro, caracterizándose estas últimas por unas condiciones laborales a menudo deficitarias y por la sobrecarga profesional.

«estábamos moviéndonos bastante con intención de fomentar el asociacionismo, pero ahora mismo estamos un poco atados. La gente que hay contratada está sobrecargada» (P.t.e.18)

3.3. La apuesta por una sociedad responsable, diversa e inclusiva: una concepción más social del éxito

A continuación, desarrollamos las propuestas referentes al fomento y promoción de avances en la sociedad en su conjunto. Cabe comenzar subrayando la importancia de una sociedad inclusiva y diseñada para todas las personas, como punto de partida en el que nuestras/os expertas/os ponen especial énfasis.

«Parece que no nos damos cuenta de que una buena sociedad es la que integra a todo el mundo. De otra manera no será una buena sociedad para vivir (...) Una sociedad para todos. Este sería el lema (risas)» (P.p.e.15)

Reivindican la pertinencia de una sociedad intercultural, que promueva una convivencia real de todos sus cohabitantes y fomente las diversas capacidades 
de los mismos, en lugar de dividir la sociedad en «vencedores» $\mathrm{y}$ «vencidos». En este sentido, destacan los perjuicios del actual sistema selectivo y meritocrático, apostando por un cambio real.

«Una cosa que realmente tienen que entender es que hay que dejar de seleccionar a la gente, por ejemplo, en el ámbito educativo, sino promover esas capacidades. Esto supondría un cambio en las competencias de la gente y supondría un gran cambio, porque nuestro sistema es muy selectivo y produce ganadores y perdedores. Es más, está interesado en producirlos. Por tanto, éste sería un cambio real» (P.p.e.16).

Además, una sociedad inclusiva tiene que ofrecer empleos para todos, es decir, incluir en su catálogo de empleos una serie de trabajos adaptados a las diferentes capacidades individuales. En este sentido, una de las analistas sociales pone de manifiesto la necesidad de inventar nuevas fórmulas de empleabilidad y de estudiar la forma en que necesidades sociales latentes puedan convertirse en oportunidades laborales. Un claro ejemplo sería lo que en la última década ha sucedido con el sistema de cuidado a la dependencia en España, que se espera continúe desarrollándose de cara al futuro:

"a la vuelta de 25 años, como mucho, tendremos un país absolutamente envejecido que necesitará cuidados y atenciones, y eso se puede convertir no solo en una cuestión de justicia y de derecho, sino oportunidades de empleo para que la gente pueda ocuparse de usar esos servicios» (P.p.c.5)

Esto está conectado con las conclusiones del Informe de Desarrollo Humano de 2014 (PNUD, 2014) que argumenta que la sostenibilidad del progreso humano pasa irrefutablemente por la reducción de vulnerabilidades y la construcción de resiliencia. Ello precisa de la identificación de los grupos estructuralmente vulnerables, así como de la adopción de un enfoque centrado en las personas. Reconoce, en definitiva, la importancia de una protección social universal, que no sólo mejora la resiliencia individual, sino que también refuerza la resiliencia de la economía en su conjunto. Además, las/os entrevistadas/os apuntan a que los cambios acaecidos en el modelo, estructura y funcionamiento familiares, requieren un cambio en los sistemas de protección, atención y cuidados. Este refuerzo de lo público conlleva y requiere, por motivos evidentes, un aumento de profesionales implicados en su correcto desarrollo.

En este sentido apuntan a la universalidad, la justicia social y la justicia redistributiva de los países nórdicos, que aparecen como referentes, si bien apuntan que la clave no estaría en "copiar el modelo», sino en analizar las potencialidades de su adaptación.

«Hacer ver que necesitamos un sector público potente para atender las realidades que tenemos. Convencer a la gente de que eso es necesario. Yo pienso en Suecia, Dinamarca, en esos países del norte de Europa donde el sector público tiene un 
peso destacadísimo. Y ¿por qué no podemos desarrollar ese modelo aquí? y además todo esto tiene que ver (no sé si me salgo del tema) con un contexto en el que la realidad familiar se está transformando en España de una manera acelerada. Y la familia que cada vez es más pequeña, y más débiles las relaciones que los miembros establecen entre ellos. No se les puede pedir que atiendan todas las contingencias como lo hacian con anterioridad, cuando las familias eran más extensas y los lazos que unían a las familias eran más fuertes» (p.p.c.12)

«La clave ha estado en el tema de redistribución de recursos públicos, la justicia redistributiva, en el pago de impuestos. En la financiación se ha apostado por un modelo de bienestar público, orientado a una justicia más distributiva y no tanto a un modelo neoliberal, como en el que estamos ahora mismo, en el que lo que importa es la rentabilidad económica (...) Una justicia social, unos derechos básicos fundamentales a todas las personas, ciudadanos y personas, y cuando digo personas no me remito solo al ciudadano, sino a españoles y no españoles» (haciendo referencia al modelo de bienestar nórdico- p.t.e.2).

Se vincula la justicia social a la justicia redistributiva y esta última al sentido de de responsabilidad social. A su vez, la responsabilidad social se relaciona con una concepción más social del éxito:

«una opción mucho mejor sería una sociedad con responsabilidad social, que no defina tanto a las personas en función de su éxito material y sus resultados profesionales, sino que los valorase por otros méritos, como la competencia social, éxito en las relaciones sociales, servicios o trabajos comunitarios. Este sería el otro dibujo de la sociedad, pero requeriría un cambio importante de mentalidad. Sería necesario repensar lo que se considera el éxito. Habría que cambiar la definición de éxito por una mucho más social» (P.p.e.4)

En una sociedad con un creciente calado y perpetuación de la teoría del merecimiento, en la que el éxito personal se equipara de manera creciente al éxito laboral, se demanda una mayor flexibilidad e incentivos a otras formas de desarrollo personal (p. e. la formación) o social (p. e. cuidados). En definitiva, se reclama el reconocimiento de diferentes estilos de vida, más allá del tradicionalmente considerado y valorado «trabajo productivo», término que hace alarde de los postulados en los que se asienta. Autores como Santos (2005) aportan luz en esta línea, proponiendo avanzar de la concepción técnica de la evaluación a la dimensión crítica de la misma. Esto es una modernización de la forzada visión dicotómica del éxito-fracaso, con fuerte carácter maniqueo, basado en interpretaciones y criterios meramente técnicos, que encuentran su raíz en construcciones sociales meritocráticas. En una sociedad cambiante, en la que las necesidades evolucionan, nuestra actual concepción de bienestar y calidad de vida dista de la de nuestros antecesores, resultando necesaria una modernización y adaptación a dichos cambios. 
«La posibilidad de dejar el mercado laboral, por asuntos de formación o cuidado, de forma que puedas vivir de ello si no trabajas, aunque, por supuesto, si quieres más tengas que trabajar... Se necesita pensar en nuevas formas de vida que no sólo sean trabajo, sino también cuidados a los otros y a uno mismo. Es parte de la vida y necesita tiempo y recursos» (P.p.c.5).

No es de extrañar, por tanto, que se reclame el reconocimiento social de distintas alternativas vitales, así como una mayor libertad en el uso del tiempo.

"Otra idea es tener tiempo para hacer algo. En cada persona hay un momento para la educación. No tienes que completarla cuando eres joven, puedes hacerlo después. También tiene que haber momentos para cuidarse a sí mismo y a los otros. Y si tú usas este tiempo para cuidar a los otros, esto debería ser compensado cuando llegas a la edad de jubilación. Tiene que haber más libertad en el uso del tiempo» (P.p.e.12)

\section{Conclusiones}

En la presente investigación nos hemos nutrido del conocimiento de campo de profesionales de Trabajo Social sobre la realidad de la intervención en servicios sociales y de sus propuestas de cambio. Entre las propuestas de intervención social con individuos y familias, nos encontramos con dos líneas de intervención a reforzar. Aunque a priori podría considerarse que ambas líneas son opuestas, tras el análisis se constatan complementarias. Por un lado, la importancia de una aproximación real al sujeto y a la situación problema. En esta línea, se defienden ideas como la necesidad de un estudio más exhaustivo de la problemática y necesidades, el acompañamiento, el apoyo subsidiario -incluso cuando no hay recursos para dar respuesta a esa problemática- y el refuerzo de la visión humanitaria. Destaca, asimismo, la importancia de la prevención, la detección temprana de los problemas y la pronta intervención, evitando el agravamiento de las situaciones problema. Por otro lado, la importancia de lo colectivo, es decir, de divisar los problemas de la sociedad en su conjunto. En esta línea, se apuesta por reforzar la intervención comunitaria pública que, relegada a un segundo plano en la actual coyuntura de crisis, podría suponer la clave para la superación de determinados problemas sociales -de lo que ya se han hecho eco algunas instituciones sociales del tercer sector-. Asimismo, la participación social y ciudadana se concibe como una clave para la superación de las adversidades, dando lugar a una mayor riqueza de recursos humanos y redes de apoyo disponibles y favoreciendo el desarrollo del tejido social, la justicia y la equidad social. Por último, se apuesta por una sociedad más responsable socialmente, inclusiva y consciente de la diversidad de talentos 
coexistentes, que reconozca distintos estilos de vida y desarrolle una concepción más social del éxito.

Se concluye la importancia de que los poderes públicos tomen conciencia de los cambios experimentados en la práctica de la intervención social desde servicios sociales y de los riesgos asociados a los mismos. Asimismo, resulta fundamental que la conciencia sobre este hecho por parte de la academia y las/os profesionales del Trabajo Social se materialice en la propuesta de medidas específicas que, desde los distintos niveles de intervención, defiendan los postulados y principios básicos de la profesión.

\section{Bibliografía}

Abad, B. y MARTín, I. (2015). El Trabajo Social ante la crisis. Nuevos retos para el ejercicio profesional de los y las trabajadoras sociales. Cuadernos de trabajo Social, 28(2), 175-185. https://doi.org/10.5209/rev_CUTS.2015.v28.n2.48765

AlguaCIL, J (2012). La quiebra del incompleto sistema de Servicios Sociales en España. Cuadernos de Trabajo Social, 25(1), 63-74. https://doi.org/10.5209/ rev_CUTS.2012.v25.n1.38434

Antón, A. (2012). Política social en tiempos de crisis. Cuadernos de Trabajo Social, 25(1), 49-62. https://doi.org/10.5209/rev_CUTS.2012.v25.n1.38433

Ávila, D. y Malo de Molina, M. (2010). Manos invisibles. De la lógica neoliberal en lo social. Trabajo social hoy, 59, 137-171 Recuperado de https://dialnet. unirioja.es/servlet/articulo?codigo $=3408143$

BAllestero, A., ÚRIZ, M.J. y VisCARET, J.J. (2012) Dilemas éticos de las trabajadoras y los trabajadores sociales en España. Papers, 97(4), 875-898. https://doi. org/10.5565/rev/papers/v97n4.283

BarRera-Algarín, E., Malagón-Bernal, J.L. y Sarasola-SánChez, J.L. (2013). La deconstrucción del Estado de bienestar: cambios en el ejercicio profesional de los trabajadores sociales y aumento del voluntariado social. Cuadernos de Trabajo Social, 26(1), 115-126. https://doi.org/10.5209/rev_CUTS.2013.v26. n1.39272

Consejo General del Trabajo Social (2019). V Foro político: Propuestas políticas desde el Trabajo Social. Recuperado de https://n9.cl/we78

De la PAz, R., Rodríguez, V. y MerCado, E. (2014). Nuevas tendencias de intervención en Trabajo Social. Azarbe. Revista Internacional de Trabajo Social y Bienestar, 3, 223-228. Recuperado de https://revistas.um.es/azarbe/article/ view/198671/161841

De LA Red, N. y BARRAnCo, C. (2014). Trabajo Social y participación en las políticas sociales. Azarbe. Revista Internacional de Trabajo Social y Bienestar, 3, 39-45. Recuperado de https://revistas.um.es/azarbe/article/view/198341/161611 
GARCíA, S. y ArÉVAlo, D. (2016). Retos en la intervención social del Trabajador/a Social. Documentos de Trabajo Social, 57, 217-226. Recuperado de https://dialnet.unirioja.es/servlet/articulo? codigo $=6095386$

García-Domingo, M. y Sotomayor, E. (2017). El rol del profesional del Trabajo Social en una coyuntura de crisis: oportunidades de la adaptación a un contexto cambiante. Trabajo Social Global-Global Social Work, 7(12), 47-58. http:// hdl.handle.net/10481/47010

GARCíA-Domingo, M. (2018). Aproximación a los cambios percibidos en los servicios sociales por sus gestores: una adaptación impuesta. Zerbitzuan, 65, 19-27. Recuperado de http://www.zerbitzuan.net/documentos/zerbitzuan/ Aproximacion_cambios_percibidos.pdf

García-Moreno, C. y Anleu-HernándeZ, C. M. (2019). Social work in Spain: a new social and economic reality to develop in practical academic training. Social Work Education, 38(8), 996-1009. https://doi.org/10.1080/0261 5479.2019.1611756

GASPAR, J-F. (2012). Tenir! Les raisons d'être des travailleurs sociaux. Paris: La Découverte.

GubA, E. y Lincoln, Y. (2002). Paradigmas en competencia en la investigación cualitativa. En C. Denman y J.A. Haro (coords.). Por los rincones. Antología de métodos cualitativos en la investigación social (pp. 113-145). Sonora: Colegio de Sonora.

Habermas, J. (1998). The Inclusion of the Other: Studies in Political Theory. Cambridge: MIT Press.

IOAKIMIDis, V., CRUZ, C. y Martínez, I. (2014). Reconceptualizing social work in times of crisis: An examination of the cases of Greece, Spain and Portugal. International Social Work, 57(4), 285-300. https://doi. org/10.1177/0020872814524967

MarChioni, M. (1999). Comunidad, participación y desarrollo. Madrid: Editorial Popular.

Morley, C. y Dunstan, J. (2013). Critical Reflection: A response to neoliberal challenges to field education. Social Work Education, 32(2), 41-56. https://doi. org/10.1080/02615479.2012.730141

NAVARro, V. (2014). El crecimiento de las desigualdades como causa de la crisis. Público. Recuperado de https://n9.cl/vgzi

Pacheco-Mangas J. y PAlma-García, M.O. (2015). Servicios Sociales, crisis y reforma local: propuestas discursivas desde el Trabajo Social. Trabajo Social Global- Global Social Work, 5(8), 47-67. Recuperado de http://hdl.handle. net/10481/36790

PELÁEZ E. y PASTOR E. (2019). Evaluación de la gestión de prestaciones económicas en servicios sociales comunitarios: el caso de Andalucía (España). Revista de 
Trabajo Social, 21(1), 39-79. Recuperado de https://dialnet.unirioja.es/servlet/ articulo? codigo $=6920850$

PRATS, J. (2005). De la burocracia al management, del management a la gobernanza. Las transformaciones de las Administraciones Públicas de nuestro tiempo. Madrid: INAP-Estudios Goberna.

PNUD (2014). Informe sobre Desarrollo Humano 2014. Sostener el progreso humano: reducir vulnerabilidades y construir resiliencia. Recuperado de http://hdr.undp. org/es/content/indice-de-pobreza-multidimensional-ipm

Pochet, P. y DeGryse, C. (2012). The programmed dismantling of the European social model. Intereconomics, 47(4), 212-217. Recuperado de https://www. econbiz.de/Record/the-programmed-dismantling-of-the-european-socialmodel-pochet-philippe/10009612601

Puig. C. (2015). Diez propuestas para cuidarse y cuidar en las profesiones sociales. Hacia la construcción de una cultura del cuidado en los profesionales. Alternativas. Cuadernos de Trabajo Social, 22, 171-183. https://doi.org/10.14198/ ALTERN2015.22.10

RIECHMAN, J. y FERNÁNDEZ-BUEY, F. (1994). Redes que dan libertad. Introducción a los nuevos movimientos sociales. Barcelona: Paidós.

Ruiz-OlabuÉNAGA, J.I. (1996). Metodología de la investigación cualitativa. Bilbao: Universidad de Deusto.

SANTOS, M.A. (2005). Evaluar es comprender. De la concepción técnica a la dimensión crítica. Revista Investigaciones en Educación, 5(1), 67-85.

SALEEBEY, D. (1996). The strengths perspective in social work practice: extensions and cautions. Social Work, 41(3), 296-305. https://doi.org/10.1093/sw/41.3.296

Smale, G., Tuson, G. y Statham, D. (2015). Problemas sociales y trabajo social: hacia la inclusión y el cambio sociales. Madrid: Morata.

STARKE, P., KAASCH, A. y VAN HOOREN, F. (2013). The welfare state as crisis manager: Explaining the diversity of policy responses to economic crisis. Londres: Palgrave Macmillan.

SzIKRA, D. (2014). Democracy and welfare in hard times: The social policy of the Orbán Government in Hungary between 2010 and 2014. Journal of European Social Policy, 24(5), 486-500. https://doi.org/10.1177/0958928714545446

TAYlOR-GoOBY, P. (2012). Beveridge Overboard? How the UK Government Is Using the Crisis to Permanently Restructure the Welfare State. Intereconomics, 47(4), 224-229. Recuperado de https://www.econbiz.de/Record/ beveridge-overboard-how-the-uk-government-is-using-the-crisis-to-permanently-restructure-the-welfare-state-taylor-gooby-peter/10009612599

Telleira, I. (2010). Recuperar la comunidad en clave democrática. En E. Martínez y L. Peña (coords.). REDefiniendo el Trabajo Comunitario (pp. 39-47). VitoriaGasteiz: Escuela Universitaria de Trabajo Social de la UPV. 
Uceda, F.X., Martínez, L., Navarro, J.J. y Botija, M.M. (2014). La pérdida de garantías de los servicios sociales comunitarios: la reforma local. Azarbe, Revista Internacional de Trabajo Social y Bienestar, 3, 247-251. Recuperado de https://revistas.um.es/azarbe/article/view/198711

Vicente-GimÉneZ, T. y BERZOSA, C. (2014). El triunfo de las finanzas capitalistas y el deterioro de los derechos sociales y de los derechos ecológicos. Jueces para la democracia, 80, 12-23. Recuperado de https://n9.cl/k5nc

Zimmerman, S. (2006). Field Education: Linking Self-Efficacy Theory and the Strengths Perspective. The Journal of Baccalaureate Social Work, 12(1), 261-274. https://doi.org/10.18084/1084-7219.12.1.261 